\title{
Determinación de la estructura molecular de geles mediante cálculos computacionales
}

\author{
M. Á. DíAZ-DíEZ , A, MACÍAS-GARCÍA, G. SILVERO'1, R,GORDILLO' Y R. CARUSO² \\ Dep. de Electrónica e Ingeniería Electromecánica. Escuela de Ingenierías Industriales. Universidad de Extremadura. 06071 Badajoz, Spain \\ ${ }^{1}$ Departamento de Química Orgánica, Facultad de Ciencias. Universidad de Extremadura. 06071 Badajoz, Spain \\ ${ }^{2}$ Laboratorio de Materiales Cerámicos, Facultad de Ciencias Exactas, Ingeniería y Agrimensura, Universidad Nacional de Rosario, (IFIR-CONICET), \\ (2000) Rosario, Argentina. Member, CONICET, Argentina.

\begin{abstract}
Este trabajo recoge un estudio dirigido al diseño de la geometría molecular de geles de $\mathrm{ZrO}_{2}$ empleando cálculos teóricos (Density-Functional Theory). En él se estudian las propiedades electrónicas y espectroscópicas (frecuencias de vibración y espectros de $\mathrm{RMN}$ de ${ }^{1} \mathrm{H}$ y ${ }^{13} \mathrm{C}$ ) de los
\end{abstract} \\ complejos de zirconio $\left[\mathrm{Zr}(\mathrm{OH})_{6}\right]^{2-}$ y $\left[\mathrm{Zr}(\mathrm{OH})_{5}\left(\mathrm{OCH}_{2} \mathrm{CH}_{2} \mathrm{CH}_{3}\right)\right]^{2-}$ \\ Palabras clave: $\mathrm{ZrO}_{2}$, Sol-Gel, estructura molecular, estudio computacional, DFT.

\section{Determination of gels molecular structure by computational calculations} \\ This work constitutes a study guided to the design of the molecular geometry of $\mathrm{ZrO}_{2}$ gels aided by computer-based calculations (Density- \\ Functional Theory). The electronic and spectroscopic properties (molecular vibrational frequencies, and ${ }^{1} \mathrm{H}$ and ${ }^{13} \mathrm{C}$ NMR spectra) of two \\ zirconium complexes, $\left[\mathrm{Zr}(\mathrm{OH})_{6}\right]^{2-}$ and $\left[\mathrm{Zr}(\mathrm{OH})_{5}\left(\mathrm{OCH}_{2} \mathrm{CH}_{2} \mathrm{CH}_{3}\right)\right]^{2-}$, are explored. \\ Key words: $\mathrm{ZrO}_{2}$, Sol-Gel, molecular structure, computational study, DFT.
}

\section{INTRODUCCIÓN}

El proceso sol-gel es uno de los métodos más importantes para obtener materiales cerámicos de base $\mathrm{ZrO}_{2}$. Mediante este proceso pueden prepararse polvos y recubrimientos con una amplia variedad de composiciones y elevada pureza [1-4].

La vía sol-gel permite la obtención de una estructura compleja de óxidos por reacciones de hidrólisis y condensación de compuestos metalorgánicos en solución. Las velocidades relativas de estas reacciones condicionan la estructura molecular de los geles y, consecuentemente, las propiedades de los materiales obtenidos [5, 6]. La simulación y diseño de estructuras mediante ordenador constituye una prometedora vía para prever las características de estos geles a nivel molecular.

Este trabajo constituye un primer estudio encaminado a la determinación de estructuras moleculares de geles de zircona mediante cálculos computacionales. El estudio se ha realizado a nivel DFT (Density-Functional Theory) [7]. Así, se han realizado cálculos que incluyen la optimización de las geometrías, determinación de las frecuencias de vibración moleculares y propiedades de RMN de estos geles $\left(\left[\mathrm{Zr}(\mathrm{OH})_{6}\right]^{2-}(\right.$ modelo 1$)$ y $\left[\mathrm{Zr}(\mathrm{OH})_{5}\left(\mathrm{OCH}_{2} \mathrm{CH}_{2} \mathrm{CH}_{3}\right)\right]^{2-}$ (modelo 2) a fin de compararlos con los espectros experimentales.

Con estos resultados se pretende en un futuro obtener geles de $\mathrm{ZrO}_{2}$ con diferentes estructuras moleculares desde soluciones sol-gel preparadas desde diferentes rutas de síntesis. A estos geles se les realizarán los espectros IR y RMN los cuales serán minuciosamente analizados a fin de encontrar las coincidencias de los espectros teóricos y experimentales.

\section{MÉTODO DE CÁLCULO}

Los cálculos teóricos, que permiten analizar la estructura y propiedades de la materia, se realizaron utilizando el paquete de programas GAUSSIAN 94 [8]. Dentro de la amplia gama de métodos para llevar a cabo este tipo de estudios teóricos, se encuentra la denominada Teoría de Funcionales de Densidad (Density-Functional Theory, DFT) [7].
Este método, basado en las Leyes de la Mecánica Cuántica, considera la interacción entre los electrones de una molécula (correlación electrónica) de forma que cada electrón interacciona con una densidad electrónica promedia representada por una función general. Así pues, el empleo de este método permite la obtención de resultados precisos para sistemas con un elevado número de átomos y con un coste computacional bajo, en comparación con otros métodos teóricos $a b$ initio que incluyen los efectos de la correlación electrónica. Este método no considera el movimiento de cada electrón individual, sino el número promedio de electrones localizados en cualquier punto del espacio.

Con estas premisas se exploraron las propiedades electrónicas y espectroscópicas de dos modelos de complejos de $\mathrm{Zr}$ (modelos 1 y 2).

En nuestro estudio se utilizó el funcional híbrido Becke3Lyp [7] junto con el conjunto de bases 6-31G (d) [9] para los núcleos de C, $\mathrm{H}, \mathrm{O}$ y Si y el conjunto de bases LANL1MB [10] para el núcleo de Zr. Las geometrías de ambos modelos fueron optimizadas, localizándose aquellas estructuras moleculares de energía más baja para cada uno de los modelos considerados. Posteriormente se calcularon sus frecuencias de vibración y se visualizaron con el programa MOLDEN [11], con objeto de asignar las bandas de los espectros de infrarrojo a los modos de vibración de las moléculas.

\section{RESULTADOS Y DISCUSIÓN}

En la Tabla 3 se muestran las estructuras optimizadas de los modelos 1 y 2, correspondientes a complejos hexacoordinados de zirconio en forma de bipirámide tetragonal.

Para el modelo 1 , se observó una carga parcial de +1.34 u.a. sobre el átomo de zirconio mientras que la carga negativa estaba igualmente distribuida entre los átomos de oxígeno. Para esta molécula se encontró un momento dipolar teórico de 2.66 debyes. El modelo 2 presentó un momento dipolar de 6.50 debyes y de nuevo, una carga parcial positiva de 1.38 u.a. sobre el núcleo metálico. El oxígeno 2 (ver Tabla 3) 
soporta una carga parcial negativa de 0.70 u.a. y los restantes átomos de oxígeno soportan una carga parcial negativa de aproximadamente 0.50 u.a.

El mayor momento dipolar del modelo 2, y en consecuencia, el aumento de la polaridad encontrada se debe a la presencia del grupo propóxido. La carga parcial negativa que soporta el oxígeno 2 en ese modelo, podrá favorecer la posible protonación y posteriormente las reacciones de hidrólisis en este núcleo, o bien, al haber moléculas en la disolución posibilitar las reacciones de condensación entre dos moléculas metal-alcóxido. Estas reacciones de condensación conducirán a largas cadenas poliméricas con tendencia a ser unidas de una forma primaria. En contraste, para el modelo 1, las reacciones de condensación tendrán lugar desde cualquiera de los grupos -OH dando lugar a cluster ramificados o en definitiva a estructuras más complejas.

En las Tablas 1 y 2 se muestran los valores calculados para los ángulos y las distancias interatómicas de los dos complejos de zirconio investigados.

TABLA 1. ÁNGULOS Y DISTANCIAS INTERATÓMICAS DEL MODELO 1

\begin{tabular}{|c|c|c|c|c|c|}
\hline \multicolumn{2}{|c|}{$\begin{array}{c}\text { Distancias } \\
\text { interatómicas }(\AA ̊)\end{array}$} & \multicolumn{4}{|c|}{ Ángulos interatómicos (Grados) } \\
\hline $\mathrm{Zr}(1)-\mathrm{O}(2)$ & 2.158 & $\mathrm{O}(2)-\mathrm{Zr}(1)-\mathrm{O}(3)$ & 89.703 & $\mathrm{O}(5)-\mathrm{Zr}(1)-\mathrm{O}(6)$ & 90.294 \\
\hline $\mathrm{Zr}(1)-\mathrm{O}(3)$ & 2.131 & $\mathrm{O}(2)-\mathrm{Zr}(1)-\mathrm{O}(4)$ & 180.000 & $\mathrm{O}(5)-\mathrm{Zr}(1)-\mathrm{O}(7)$ & 89.712 \\
\hline $\mathrm{Zr}(1)-\mathrm{O}(4)$ & 2.162 & $\mathrm{O}(2)-\mathrm{Zr}(1)-\mathrm{O}(5)$ & 89.764 & $\mathrm{Zr}(1)-$ & 100.923 \\
\hline $\mathrm{Zr}(1)-\mathrm{O}$ & 2.187 & $(2)-\mathrm{Zr}(1)-\mathrm{O}(6)$ & 90.000 & $\operatorname{Zr}(1)-$ & 99.315 \\
\hline $\mathrm{Zr}(1)-\mathrm{O}(6)$ & 2.134 & $\mathrm{O}(2)-\mathrm{Zr}(1)-\mathrm{O}(7)$ & \begin{tabular}{|l|}
89.790 \\
\end{tabular} & $\mathrm{Zr}(1)-\mathrm{O}(4)-\mathrm{H}(11)$ & 101.352 \\
\hline $\mathrm{Zr}(1)-\mathrm{O}(7)$ & 2.188 & $\mathrm{O}(3)-\mathrm{Zr}(1)-\mathrm{O}(4)$ & 90.534 & $\mathrm{Zr}(1)-\mathrm{O}(5)-\mathrm{H}(10)$ & 98.827 \\
\hline $\mathrm{O}(2)-\mathrm{H}(13)$ & 0.971 & $\mathrm{O}(3)-\mathrm{Zr}(1)-\mathrm{O}(5)$ & 179.451 & $\mathrm{Zr}(1)-\mathrm{O}(6)-\mathrm{H}(8)$ & 101.560 \\
\hline $\mathrm{O}(3)-\mathrm{H}(12)$ & 0.971 & $\mathrm{O}(3)-\mathrm{Zr}(1)-\mathrm{O}(6)$ & 89.731 & $\mathrm{Zr}(1)-\mathrm{O}(7)-\mathrm{H}(9)$ & 100.932 \\
\hline $\mathrm{O}(4)-\mathrm{H}(11)$ & 0.972 & $\mathrm{O}(3)-\mathrm{Zr}(1)-\mathrm{O}(7)$ & 90.261 & $\mathrm{Zr}(1)-\mathrm{O}(2)-\mathrm{H}(13)$ & 100.923 \\
\hline $\mathrm{O}(5)-\mathrm{H}(10)$ & 0.971 & $\mathrm{O}(4)-\mathrm{Zr}(1)-\mathrm{O}(5)$ & 89.999 & $\mathrm{Zr}(1)-\mathrm{O}(3)-\mathrm{H}(12)$ & 99.315 \\
\hline $\mathrm{O}(6)-\mathrm{H}(8)$ & 0.972 & $\mathrm{O}(4)-\mathrm{Zr}(1)-\mathrm{O}(6)$ & 90.000 & $\mathrm{Zr}(1)-\mathrm{O}(4)-\mathrm{H}(11)$ & 101.352 \\
\hline $\mathrm{O}(7)-\mathrm{H}(9)$ & 0.972 & $\mathrm{O}(4)-\mathrm{Zr}(1)-\mathrm{O}(7)$ & 90.207 & $\mathrm{Zr}(1)-\mathrm{O}(5)-\mathrm{H}(10)$ & \begin{tabular}{|l|}
98.827 \\
\end{tabular} \\
\hline & & & & $\mathrm{Zr}(1)-\mathrm{O}(6)-\mathrm{H}(8)$ & 101.560 \\
\hline
\end{tabular}

Como puede observarse, los parámetros estructurales de ambos complejos muestran la geometría octaédrica esperada, solo distorsionada por la presencia del grupo propóxido en el modelo 2.

TABLA 2. ÁNGULOS Y DISTANCIAS INTERATÓMICAS DEL MODELO 2

\begin{tabular}{|c|c|c|c|c|c|}
\hline \multicolumn{2}{|c|}{$\begin{array}{c}\text { Distancias } \\
\text { interatómicas ( }(\AA)\end{array}$} & \multicolumn{4}{|c|}{$\begin{array}{c}\text { Ángulos interatómicos } \\
\text { (Grados) }\end{array}$} \\
\hline $\mathrm{Zr}(1)-\mathrm{O}(2)$ & 2.201 & $\mathrm{O}(2)-\mathrm{Zr}(1)-\mathrm{O}(3)$ & \begin{tabular}{|l|}
89.701 \\
\end{tabular} & $\mathrm{Zr}(1)-\mathrm{O}(4)-\mathrm{H}(11)$ & 101.731 \\
\hline $\mathrm{Zr}(1)-\mathrm{O}(3)$ & 2.119 & $\mathrm{O}(2)-\mathrm{Zr}(1)-\mathrm{O}(4)$ & 180.000 & $\mathrm{Zr}(1)-\mathrm{O}(5)-\mathrm{H}(10)$ & 102.476 \\
\hline $\mathrm{Zr}(1)-\mathrm{O}(4)$ & 2.125 & $\mathrm{O}(2)-\mathrm{Zr}(1)-\mathrm{O}(5)$ & \begin{tabular}{|l|}
89.766 \\
\end{tabular} & $\mathrm{Zr}(1)-\mathrm{O}(6)-\mathrm{H}(8)$ & 102.411 \\
\hline $\mathrm{Zr}(1)-\mathrm{O}(5)$ & 2.182 & $\mathrm{O}(2)-\mathrm{Zr}(1)-\mathrm{O}(6)$ & \begin{tabular}{|l|}
90.000 \\
\end{tabular} & & 102.873 \\
\hline $\mathrm{Zr}(1)-\mathrm{O}$ & 2.128 & $\mathrm{O}(2)-\mathrm{Zr}(1)-\mathrm{O}(7)$ & \begin{tabular}{|l|}
89.790 \\
\end{tabular} & $\mathrm{O}(2)$ & 112.235 \\
\hline $\mathrm{Zr}(1)$ & 2.1 & $\mathrm{O}(3$ & 90.541 & $\mathrm{O}($ & 1 \\
\hline $\mathrm{O}(2)-\mathrm{C}$ & 1.3 & $\mathrm{O}(3)-$ & 179.451 & & \\
\hline $\mathrm{O}(3)-\mathrm{H}(12)$ & 0.970 & $\mathrm{O}(3)-\mathrm{Zr}(1)-\mathrm{O}(6)$ & 89.731 & -H(15) & 107.398 \\
\hline $\mathrm{O}(4)-\mathrm{H}(11)$ & 0.971 & $\mathrm{O}(3)-2$ & 90.261 & $\mathrm{C}(14$ & 105.896 \\
\hline $\mathrm{O}(5)-\mathrm{H}(10)$ & 0.971 & $\mathrm{O}(4)-$ & 89 & $\mathrm{H}(15$ & 105.546 \\
\hline $\mathrm{O}(6$ & 0.971 & $\mathrm{O}(4$ & & & 01 \\
\hline $\mathrm{O}(7)$ & 0.972 & $\mathrm{O}(4)$ & 90.208 & 8) & \\
\hline$C(13)-C(14)$ & 1.551 & $\mathrm{O}(5)-\mathrm{Zr}(1)-\mathrm{O}(6)$ & \begin{tabular}{|l|}
90.298 \\
\end{tabular} & $-\mathrm{H}(119)$ & 108.026 \\
\hline $\mathrm{C}(13)-\mathrm{H}(15)$ & 1.112 & $\mathrm{O}(5)-\mathrm{Zr}(1)-\mathrm{O}(7)$ & \begin{tabular}{|l|}
89.708 \\
\end{tabular} & $\mathrm{C}(17)-\mathrm{C}(14)-\mathrm{H}(18)$ & 110.133 \\
\hline $\mathrm{C}(13)-\mathrm{H}(16)$ & 1.123 & $\mathrm{O}(6)-\mathrm{Zr}(1)-\mathrm{O}(7)$ & 180.000 & $\mathrm{C}(17)-\mathrm{C}(14)-\mathrm{H}(19)$ & 109.947 \\
\hline$C(14)-C(17)$ & 1.534 & $\mathrm{Zr}(1)-\mathrm{O}(2)-\mathrm{H}(13)$ & \begin{tabular}{|l|}
129.148 \\
\end{tabular} & $\mathrm{H}(18)-\mathrm{C}(14)-\mathrm{H}(19)$ & 106.321 \\
\hline $\mathrm{C}(14)-\mathrm{H}(18)$ & 1.100 & $\mathrm{O}(2)-\mathrm{Zr}(1)-\mathrm{O}(3)$ & \begin{tabular}{|l|}
89.701 \\
\end{tabular} & $\mathrm{C}(14)-\mathrm{C}(17)-\mathrm{H}(20)$ & 112.960 \\
\hline $\mathrm{C}(14)-\mathrm{H}(19)$ & 1.099 & $\mathrm{O}(2)-\mathrm{Zr}(1)-\mathrm{O}(4)$ & 180.000 & & 110. \\
\hline $\mathrm{C}(17)-\mathrm{H}(20)$ & 1.101 & $\mathrm{O}(2)-\mathrm{Zr}(1)-\mathrm{O}(5)$ & \begin{tabular}{|l|}
89.766 \\
\end{tabular} & $\mathrm{C}(14)-\mathrm{C}(17)-\mathrm{H}(22)$ & 110.939 \\
\hline $\mathrm{C}(17)-\mathrm{H}(21)$ & 1.100 & $\mathrm{O}(2)-\mathrm{Zr}(1)-\mathrm{O}(6)$ & \begin{tabular}{|l|}
90.000 \\
\end{tabular} & $\mathrm{H}(20)-\mathrm{C}(17)-\mathrm{H}(21)$ & 107.543 \\
\hline $\mathrm{C}(17)-\mathrm{H}(22)$ & 1.100 & $\mathrm{O}(2)-\mathrm{Zr}(1)-\mathrm{O}(7)$ & \begin{tabular}{|l|}
89.790 \\
\end{tabular} & $\mathrm{H}(20)-\mathrm{C}(17)-\mathrm{H}(22)$ & 107.479 \\
\hline & & $\mathrm{Zr}(1)-\mathrm{O}(3)-\mathrm{H}(12)$ & 100.100 & $\mathrm{H}(21)-\mathrm{C}(17)-\mathrm{H}(22)$ & 106.881 \\
\hline
\end{tabular}

El grupo alquílico no afecta al valor de la distancia entre el Zr y los átomos de oxígeno, siendo éste de aproximadamente 2,15 Å. La Tabla
3 recoge las bandas más significativas en cada caso y los correspondientes modos de vibración. La principal diferencia observada entre los espectros IR de ambos modelos fue la importante ausencia de frecuencias de vibración entre 700 y $3500 \mathrm{~cm}^{-1}$ encontrada en el espectro del complejo 1, atribuible a la cadena alquílica del modelo 2 . El resto de frecuencias de vibración en ambos espectros son debidas a posibles modos de vibración simétricas, asimétricas, fuera del plano y en el plano de los distintos enlaces $\mathrm{Zr}-\mathrm{O}$, O-H y C-O (Tabla 3).

TABLA 3. BANDAS Y MODOS DE VIBRACIÓN EN LOS ESPECTROS TEÓRICOS DE LOS MODELOS 1 Y 2

\begin{tabular}{|c|c|c|}
\hline Compound & $\mathrm{cm}^{-1}$ & $\begin{array}{l}\text { Modos de } \\
\text { vibración }\end{array}$ \\
\hline & 384 & $\begin{array}{c}\text { Simétrica } \\
\mathrm{Zr}-\mathrm{O}\end{array}$ \\
\hline & 420 & $\begin{array}{c}\text { Asimétrica } \\
\text { Zr-O }\end{array}$ \\
\hline & 430 & \multirow{3}{*}{$\begin{array}{l}\text { Fuera del } \\
\text { plano } \mathrm{OH}\end{array}$} \\
\hline & 435 & \\
\hline & 512 & \\
\hline & 619 & \multirow{6}{*}{$\begin{array}{c}\text { En el plano } \\
\text { OH }\end{array}$} \\
\hline & 649 & \\
\hline & 651 & \\
\hline & 662 & \\
\hline & 671 & \\
\hline & 673 & \\
\hline & 409 & \\
\hline & 411 & Asimétrica \\
\hline & 475 & Zr-O \\
\hline & 382 & \\
\hline & 431 & Fuera del \\
\hline & 486 & plano $\mathrm{OH}$ \\
\hline & 531 & \\
\hline & 529 & \\
\hline & 648 & En el plano \\
\hline & 664 & \\
\hline & 765 & \\
\hline & 1205 & $\begin{array}{c}\text { Simétrica } \\
\mathrm{C}-\mathrm{O}\end{array}$ \\
\hline
\end{tabular}

Los tensores de desplazamiento de RMN constituyen otra propiedad que puede ser abordada computacionalmente [12]. Las constantes de desplazamiento aportadas en estudios experimentales son generalmente señales relativas a compuestos estándar, a menudo tetrametilsilano (TMS). Para predecir las señales de cada átomo es necesario restar el valor absoluto de la molécula de referencia. Las Tablas 4 y 5 muestran las señales químicas $(\delta)$ en ppm para carbono e hidrógeno en los modelos 1 y 2.

TABla 4. SeÑales QUímicas ( $\Delta$ ppm) EN MOdelo 1.

\begin{tabular}{|c|c|c|c|c|c|}
\hline H8 & H9 & H10 & H11 & H12 & H13 \\
\hline 1.68 & -0.47 & 0.19 & 0.68 & 2.14 & 0.83 \\
\hline
\end{tabular}

TABLA 5. SEÑALES QUÍMICAS ( $\triangle$ PPM) EN MODELO 2. \begin{tabular}{|l|l|l|l|l|l|l|l|l|l|l|l|l|l|l|}
\hline H8 & H9 & H10 & H11 & H12 & H15 & H16 & H18 & H19 & H20 & H21 & H22 & C13 & C14 & C17 \\
\hline
\end{tabular} \begin{tabular}{|l|l|l|l|l|l|l|l|l|l|l|l|l|l|l|}
\hline 1.24 & 1.17 & -0.13 & -0.09 & 2.39 & 5.29 & 4.12 & 0.95 & 1.03 & 0.05 & 0.57 & 0.41 & 67.1 & 30.2 & 7.39 \\
\hline
\end{tabular}

De acuerdo con las señales químicas que aparecen en la tabla 4, los protones $\mathrm{H} 8$ y $\mathrm{H} 12$ en el modelo 1 son los más desapantallados, apareciendo a campo bajo (altos valores de $\delta$ ). Este hecho es debido a la ma- 
yor densidad electrónica en este área como previamente se mostró con los vectores de los momentos dipolares en la figura 1. Por otra parte, los protones $\mathrm{H} 9$ y $\mathrm{H} 10$ aparecen a campo alto (bajos valores de $\delta$ ). En el caso del modelo 2 (Tabla 5), H15 y H16, junto al C13, directamente enlazados al O2, son los más desapantallados al igual que el C13.

\section{CONCLUSIONES}

En este trabajo a través de un estudio computacional hemos calculado la distribución de la carga parcial, el momento dipolar y las estructuras optimizadas de dos complejos de zirconio: $\left[\mathrm{Zr}(\mathrm{OH})_{6}\right]^{2-}$ $y\left[\mathrm{Zr}(\mathrm{OH})_{5}\left(\mathrm{OCH}_{2} \mathrm{CH}_{2} \mathrm{CH}_{3}\right)\right]^{2}$, teóricamente. El estudio computacional permitió también obtener los espectros de infrarrojo y los tensores de desplazamiento de RMN teóricos de ambos complejos. Esta información podrá ser utilizada a la hora de realizar predicciones sobre las reacciones de hidrólisis y condensación más favorables que tienen lugar durante el proceso sol-gel de formación de dichos complejos.

\section{BIBLIOGRAFÍA}

1. C. J Brinker and G. W. Scherer, Sol-Gel Science: The physics and chemistry of sol-gel processing, Academic Press, San Diego 1990.

2. M. Atik and M. A. Aegerter, in Proc. Sixth International Workshop on Glasses and Ceramics from Gels, Seville 1991, J. Non-Cryst. Solids, 1992; 147-148, 813-819.
3. B. J. J. Zelinski and D. R. Uhlmann, "Gel technology in ceramics", J. Phys. Chem. Solids, 1984; 45 [10] 1069.

4. J. Livage, M. Henry and C. Sanchez, Prog. Solid State Chem.1988; 18: 259.

5. B. E. Yoldas, J. Non-Cryst. Solids, 1984; 63: 145.

6. B. E. Yoldas, Material Research Society Symposium Proceeding, Elsevier Science, New York, 1984; 24: 291.

7. a) Kohn, W.; Sham, L. J.; Physical Review 1965; 140, A1133. b) Slater, J. C. Quantum Theory of Molecular and Solids; McGraw-Hill: New York, 1974. c) Vosko, S. H.; Wilk, L., Nusair, M.; Canadian J. Phys. 1980, 58, 1200. d) Lee, C. Yang, W.; Parr, R. G.; Physical Review B 1988; 37: 785-789. e) Becke, A. D.; Phys. Rev. A 1988; 38: 3098. f) Becke, A. D.; J. Chem. Phys.1993; 98: 1372. g) Parr, R. G.; Yang, Density-funtional theory of atoms and molecules; Oxford Univ. Press: Oxford, 1989.

8. Frisch, M. J.; Trucks, G. W.; Schlegel, H. B.; Gill, P. M. W.; Johnson, B. G.; Robb, M. A.; Cheeseman, J. R.; Keith, T. A.; Petersson, G. A., Montgomery, J. A.; Raghavachari, K.; Al-Laham, M. A.; Zakrzewski, V. G.; Ortiz, J. V.; Foresman, J. B.; Cioslowski, J.; Stefanov, B. B.; Nanayakkara, A.; Challacombe, M.; Peng, C. Y.; Ayala, P. Y.; Chen, W.; Wong, M. W.; Andres, J.L.; Replogle, E. S.; Gomperts, R.; Martin, R. L.; Fox, D. J.; Binkley, J. S.; Defrees, D. J.; Baker, J.; Stewart, J. P.; Head-Gordon, M.; González, C.; Pople, J. A.; Gaussian, Inc. Pittsburgh PA, 1995.

9. W.J. Hehre, L. Radom, P.v.R. Schleyer, J.A. Pople, “Ab initio molecular orbital theory", Wiley, New York, 1986.

10. (a) P.J. Hay, W.R. Wadt, J. Chem. Phys. 82 (1985) 270; (b) P.J. Hay, W.R. Wadt, J. Chem. Phys. 8 (1985) 284; (c) P.J. Hay, W.R. Wadt, J. Chem. Phys. 82 (1985) 299.

11. Schaftenaar, G.; Noordik, J. H. J. Comput.-Aided Mol. Des. 2000; 14: 123-134.

12. Cheeseman, J. R.; Trucks, G. W.; Keith, T. A.; Frisch, M. J. J. Chem. Phys.1996; 104: 5497.

Recibido: 1.2.03

Aceptado: 30.11 .03 